\title{
Evaluating Video as a Technology for Informal Communication
}

\author{
Robert S. Fish, Robert E. Kraut, Robert W. Root \\ Bellcore \\ 445 South Street, Morristown, N.J. 07962-1910 \\ robf@bellcore.com \\ $\&$ \\ Ronald E. Rice \\ SCILS, Rutgers University \\ 4 Huntington Street, New Brunswick, N.J. 08903
}

\begin{abstract}
Collaborations in organizations thrive on communication that is informal because informal communication is frequent, interactive, and expressive. Informal communication is crucial for the coordination of work, learning an organization's culture, the perpetuation of the social relations that underlie collaboration, and, in general, any situation that requires communication to resolve ambiguity. Informal communication is traditionally mediated by physical proximity, but physical proximity cannot mediate in geographically distributed organizations. The research described here evaluates the adequacy of a version of a desktop video/audio conferencing system for supporting informal communication in a research and development laboratory. The evaluation took place during a trial in which the system was used by summer employees and their supervisor-mentors. While the system was used frequently, the most common uses and users' assessments suggest that it was used more like a telephone or electronic mail than like physically mediated face-to-face communication. However, some features of its use transcended traditional media and allowed users to gain awareness of their work environment. The paper concludes with a discussion of requirements for successful technology to support informal communication.
\end{abstract}

KEYWORDS: Informal meetings, evaluation, video, desktop videoconferencing, group work, collaboration.

\section{INTRODUCTION: INFORMAL COMMUNICATION}

Communication in organizations can be more or less formal [2]. Formal communication goes through organizational

Permission to copy without fee all or part of this material is granted provided that the copies are not made or distributed for direct commercial advantage, the ACM copyright notice and the title of the publication and its date appear, and notıce is given that copying is by permıssion of the Association for Computing Machinery. To copy otherwise, or to republish, requires a too and/or specific permission.

(C) 1992 ACM 0-89791-513-5/92/0005-0037 1.50 channels following the hierarchy of an organization's structure. In contrast, informal communication cuts across these organizational boundaries. Operationally, informal communication differs from formal communication in its greater frequency, expressiveness and interactivity $[6,16,29]$. These attributes give organizational members the flexibility to deal with highly uncertain and ambiguous topics, tasks, and decisions. Informal communication is especially important for the less directly task-oriented aspects of organizational membership, for example, learning the organizational culture, becoming loyal to an organization, making judgments of others, and forming relationships.

Typically, informal communication is mediated by physical proximity. The chance encounters and ease of access among people who are physically close to each other in an organization provide many opportunities for organizational members to come into contact and communicate [21]. When people are physically close to each other, communication typically occurs through face-to-face conversations or meetings. Compared to other communication channels, face-to-face communication is socially oriented and rich. According to social presence theorists $[14,28]$ visually-oriented, face-to-face communication supports informal communication because it highlights the other people in an interaction and consequently interpersonal relationships in general. From a media richness perspective [7], visually-oriented face-to-face communication is a rich medium that is interactive (providing opportunity for timely feedback and the tailoring of messages to personal circumstances) and expressive (having the ability to convey multiple cues and to use language variety). Hence, it should be useful to increase understanding and reduce the amount of equivocality in a given communication situation.

\section{Potential of Video/Audio Technology for Informal Communication}

Both analyses of the ways the visual channel is used to support informal interaction in face-to-face settings and analyses of early experiments using video to support distributed work 
groups suggest that video communication systems might provide a basis for informal communication at a distance. Below we review the reasoning based on the prior literature that suggests that a visual channel in general and video in particular 1) is helpful in increasing the spontaneity and frequency of communication, 2) helpful in supporting social relationships, 3 ) helpful in coping with the most complex and equivocal communication problems encountered in work groups, and as a result 4 ) helpful in integrating members into and supporting the work in research and development groups.

Frequency and spontaneity of interaction. Close observation of spontaneous, face-to-face communication episodes led Kraut, Fish, Root and Chalfonte [16] to conclude that the visual channel is important in initiating informal communication. The visual channel increases the probability of informal interaction by helping organizational members simultaneously identify a partner, topic, and moment for conversation and by helping them manage the transition from lack of engagement to engagement in interaction. Recent studies of the use of video conferencing systems in R\&D laboratories show that they can lead to spontaneous interaction, although less than in face-toface settings [9]. A recent demonstration project at Xerox PARC provided a continuous video and audio connection between two of its research facilities located hundreds of miles apart $[1,10]$. Usage data indicated that over seventy per cent of the interpersonal communication between the two sites consisted of short, casual, "drop-in" interactions and that most of these interactions would not have occurred in the absence of a continuous video link.

Richness and social-orientation of interaction. Both media richness theorists [7] and social presence theorists $[14,28]$ array communication channels along a continuum anchored by faceto-face interaction at the richer, social end and written documents at the other. To the extent that audio/video communication mimics the features of face-to-face communication in being expressive, interactive, and focusing attention on personal attributes, it should function like face-toface communication. Thus, the media richness and social presence perspectives suggest that video teleconferencing should be well suited for informal communication, and especially good for aiding the more social, the more uncertain, and the more equivocal aspects of communication. For example, the evidence from early studies of video conferencing suggest that video conferences, face-to-face meetings or written exchanges are roughly equivalent for information transfer tasks, but are differentiated when consensus formation and conflict are at issue [28].

Support for research and development environments. Early trials of telemedicine [24] attempted to use video for medical information transfer, diagnosis, consultation, and patient contact. While the technology had only mixed success in supporting information transfer and diagnosis tasks, Rockoff
[25] offers a "clinical impression ... that this technology improves the cohesiveness and sense of organizational unity experienced by health care providers in a geographically dispersed system, i.e., it facilitates their functional integration (Pp 1087)." This observation is consistent with the hypothesis that at least some of the effects of the technology were in aiding the formation of social relationships and other outcomes associated with informal communication.

More recent studies of video networks in $R \& D$ organizations $[1,4,9,10]$ have illustrated the value of video communication networks, as well as showing some of their limitations. In the Xerox PARC experiment described earlier, participants' experiences suggest that having this video link was adequate to promote a shared context and culture that supported joint work across the two R\&D locations, but just barely. In discussing this experience, Olson and Bly [22], among others, note the crucial importance of having a shared work space in which participants in a discussion could jointly view and manipulate the objects in their world.

\section{Purpose of current study}

The present research examines in a less anecdotal manner than previous studies the usefulness of audio/video conferencing for the support of informal communication in a realistic setting. We were interested in the degree to which visual communication could attain the characteristics of frequency, expressiveness, and interactivity that are the hallmarks of informal communication.

We addressed this question in the context of a four week field trial in which temporary employees and their supervisor/mentors at Bellcore used a new audio/video conferencing technology called the CRUISER ${ }^{T M 1}$ system $[9$, 26]. Since it supports standard videoconferencing, the CRUISER system should have the attributes discussed above that make it appropriate for rich communication with social presence. In addition, this system included features designed to use video to increase the opportunities for communication and thus increase the frequency of spontaneous conversation. These features included system-initated calls, brief, self-terminating calls, and call sequences, all designed to simulate the experience of walking down a hallway, viewing others as they work in their offices, and taking advantage of a random contact to have a conversation. These features are described in more detail below.

\section{METHODS}

The experiment was conducted as part of the summer internship program for college and graduate students at Bellcore. Students in telecommunications engineering, computer science, mathematics, psychology, statistics, and other disciplines worked for 10 weeks with senior researchers in their field. From a pool of about 50 volunteers, we randomly selected 23

${ }^{1}$ Cruiser is a trademark and service mark of Bellcore. 
volunteers --11 students and their 12 mentors -- to use the CRUISER system.

The CRUISER environment in the summer of 1990 consisted of a software controlled audio and video telecommunications network of 30 nodes. The nodes were distributed over four wings and two floors of a large R \& D facility. Each user was equipped with a multi-windowed computer terminal that controlled the CRUISER application and provided conventional computing including $c$ electronic mail. In addition to the computer terminal, the CRUISER station consisted of a 12inch color video monitor, a small speaker, and a microphone. Audio connections were full duplex. This allowed both parties to a conversation to talk at the same time with no audio echo or feedback.

This version of the CRUISER application used three novel calling methods to encourage spontaneous conversation. These methods were:

- Cruises, in which users initiated one or a series of audio/video calls. They differed from conventional telephone-like calls in three ways. First, when the caller issued the command, the system opened an immediate audio and video connection to the called party, which timed out after about three seconds unless one party explicitly continued it by issuing a "Visit" command. During the connection, both parties could see and hear each other. Second, if users supplied a list of names (e.g., 'Cruise john, alice, mark'), the system stepped through each in turn, stopping only if one party issued the visit command. Finally, if the user supplied no name (i.e., 'Cruise'), the system selected a called party at random from among the users currently logged into the system.

- Autocruises, in which the the system itself initiated calls between selected users at random times. Except for initiation, the protocol was the same as a Cruise. A connection was made and timed out if neither party issued a Visit command. The intended analogy for the Autocruise was wandering in a corridor and seeing other people, with whom one could speak or not.

- Glances., which were very brief video-only connections to one or a series of other people. When users issued a Glance they received an approximately one second glance into the called party's office; this Glance could not be converted into a sustained connection without subsequently issuing a Cruise command. If users issued the Glance command without an argument, the system initiated a series of five brief glances of randomly selected logged-in users on the system.

The system included two features to deal with privacy concerns. First, users could issue a "private" command. This caused all others who tried to call them to see a notification that they were busy. Second, the system imposed a reciprocity rule on all calls. Users were assured that if someone else could see and hear them, they could see and hear that person as well.

Finally, to increase awareness of the other CRUISER users, the system included one feature that showed users an active directory listing the availability status of all other users and a second feature that maintained a list showing users a history of who called them.

\section{RESULTS}

\section{System usage}

There were a maximum of 23 users, both interns and mentors, on any day. During the 21 business days of the trial, users made 1295 call attempts to 1556 recipients $^{2}$ or about 2.7 call attempts per potential user per day. As with most new communication facilities introduced into organizations, users showed an early burst of activity as they tried out the system. Figure 1 shows the distribution of call attempts per potential user per day ${ }^{3}$, both over all users and separately for the 5 dropouts, users who attempted two or fewer calls during the second week of the trial, and for the 18 sustained users, who attempted more than two calls during the second week of the trial. Throughout the trial, the sustained users were placing 4 to 5 calls per business day, with call frequency understandably dropping on the weekends (days $6 \& 7,13 \& 14,20 \& 21$ ). These numbers are comparable to the frequency distribution for telephone usage whose mode is 2 outgoing calls and mean is 4 outgoing calls per user per day [20].

As described previously, calls can be either cruises or glances initiated by callers or autocruises initiated by the system, calls can be placed to one or more people simultaneously, and the name of the called party can be supplied by the caller or supplied by the system. Table 1 shows the distribution of call attempts across these call types and the percentage of each call type that were accepted. As can be seen, the modal use of the system (1015 attempts) was one in which a user intentionally placed a call to one other named party.

\begin{tabular}{|l|l|l|l|}
\hline Call type & $\begin{array}{l}\text { \# of called } \\
\text { parties }\end{array}$ & $\begin{array}{l}\% \text { calls } \\
\text { accepted }\end{array}$ \\
\hline & 1 & $>1$ & \\
\hline Cruise - caller supplied name & 1015 & 8 & $54 \%$ \\
\hline Cruise - system supplied name & 67 & NA & $18 \%$ \\
\hline Glance- caller supplied name & 174 & 9 & NA \\
\hline Glance - system supplied name & NA & 51 & NA \\
\hline Autocruise - system supplied name & 236 & NA & $3 \%$ \\
\hline
\end{tabular}

2 Some call attempts were to a series of recipients.

3 Because of uncertainty about who was present on a given day, the total number of calls per day was uniformly divided by 23 , the maximum number of users. Thus, Figure 1 underestimates per person usage on a typical day. 


\section{Use over time}

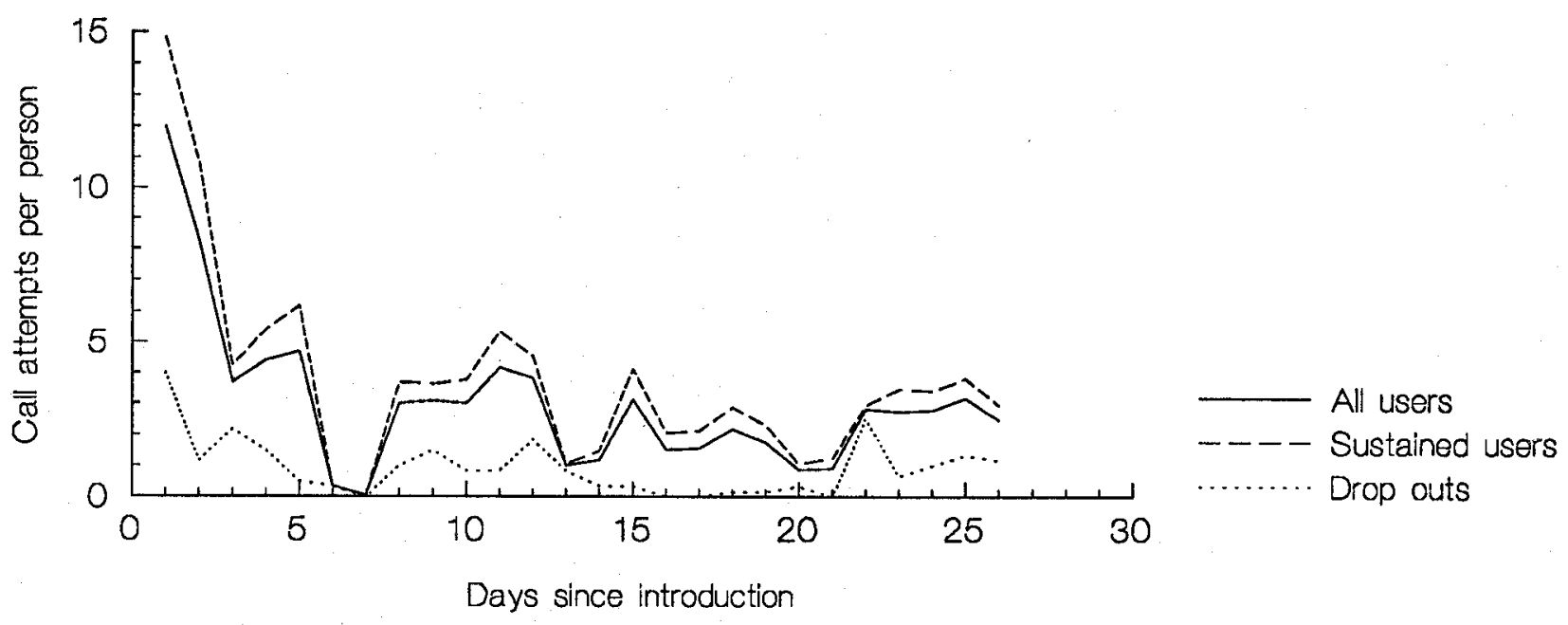

Figure 1. Number of calls over days.

Table 1. Frequency and outcomes of call attempts

However, about a quarter of user-initiated calls seem to be cases in which people are monitoring their environment rather than communicating with particular individuals. In about half of these calls, callers made a quick glance to a party they named, often to see if that person was available before placing a cruise call or visiting in person. Other calls served a more generalized awareness function. In these cases, users allowed the system to connect them to each other with the possibility for conversation (cruise) or to give them a quick, purely informational peek into the offices of people (glance).

Hence, while the most frequent use of the system was in a mode that was something like a telephone call, there was also significant use that was quite different. While telephone users may call a business to see whether they are open, individuals rarely call each other just to stay aware of who could be contacted, if and when they wanted to. We believe that the Glance mode was often chosen because users perceived that it was quicker and less intrusive than a Cruise call, and therefore provided a better balance between convenience to themselves and the degree to which the call recipients were disturbed.

Figure 2 shows the distribution of Cruise calls and Glances over time. These data substantiate the hypothesis that the two types of calls were used for different functions, since they have distinctive distributions over time (Pearson $\chi^{2}=21.08, d f=$ $11, p<.05)$. Glances were especially likely to be performed early in the morning, right after lunch, and on weekends, when uncertainty about who was around was highest and perhaps before the callers themselves had become fully absorbed in their work.

CRUISER calls were typically short. The modal CRUISER call was under 5 seconds, and the median call lasted 62 seconds. Approximately $25 \%$ lasted less than 30 seconds, $25 \%$ lasted 3 minutes or longer, and 5\% lasted more than 30 minutes. The longest calls were over 4 hours. This distribution of call duration is comparable to that observed in telephone calls [20], where the modal call is less than 30 seconds, the mean is about 4 minutes, and the distribution has a similar long tail. Long CRUISER calls include both sustained work sessions similar to those conducted by telephone and two interesting behavioral innovations that we believe were specifically enabled by this technology.

The first of these innovations might be called the virtual shared office. On occasion some intern-mentor pairs connected their offices for an extended period, without engaging in sustained conversation. Rather, the pair would work relatively independently, occasionally having conversation to ask a question or to get help on a problem. The open connection reduced the behavioral cost of communication during periods when the participants anticipated they would need multiple episodes of unscheduled conversation. Other researchers studying the use of other desktop conferencing systems have reported similar phenomena $[3,11,19]$. 
The second innovative use of long calls might be called the ambush. Here one member of a work team with a pressing communication need makes a connection to the other's office. If they are not there, instead of disconnecting and trying again later, they simply maintain the connection and wait for them to come to their office. The "waiter" can monitor the other's office through peripheral awareness, but is still able to focus on his or her own work. This is a nice example of where the technology effectively permitted people to be in two places at the same time.

\section{Comparisons to other media}

We have seen, based on system logs, that the typical CRUISER call was short and this finding was confirmed in our self-report data. In the debriefing interviews, subjects estimated the length of their last CRUISER call and the length of their last face-to-face conversation with the same partner. They reported having about 4 minute CRUISER calls versus having about 30 minute face-to-face conversations.

Not only did the conversations differ in length, but they also differed in content and outcome. During our interview, users compared a recent CRUISER conversation with a face-to-face conversation with the same partner. In Table 2 we summarize the length, content, and outcomes in their reports of the conversations.

Conversations using the CRUISER system involved more greeting and scheduling, but involved less problem solving and decision making. Of the 23 student-mentor pairs, most reported using CRUISER conversations to inquire about or to inform each other about the status of work activities, to get quick answers to short questions, or to schedule work. In essence, they reported that during CRUISER conversations they mostly prepared for work, while during face-to-face conversations they actually performed the work.

Why was this? Time and again users said that they used faceto-face communication rather than the CRUISER system because it wasn't able to support all the communication demands of conventional work activities. From a media richness perspective [7], this version of desktop teleconferencing was still insufficiently rich compared to face to face communication. For instance, a major problem that 90 per cent of respondents mentioned was that they could not share work objects when conversing with someone by using the system. One mentor, for example, called a student just as she was in the midst of trying to understand and solve a problem in a computer program she was writing. Taking advantage of this opportunity, the student asked questions and sought advice. However, after some brief discussion, the mentor walked to the student's office so that they could jointly work at the computer terminal and examine printouts. Similarly, one student reported that he often called his mentor whenever he was stuck on a problem. By using CRUISER, he could quickly tell if his mentor was available for assistance. They would briefly discuss the problem and then schedule an immediate or deferred time to meet, mainly so that they could jointly use a blackboard. In a third case, a student had a virtual office connection to his mentor while working on an outline. The student noticed the mentor at his desk and opportunistically asked him a question about the outline. This brief question led to an extended discussion. The conversation progressed to the point where the mentor asked the student to come to his office so that they could jointly use the blackboard.

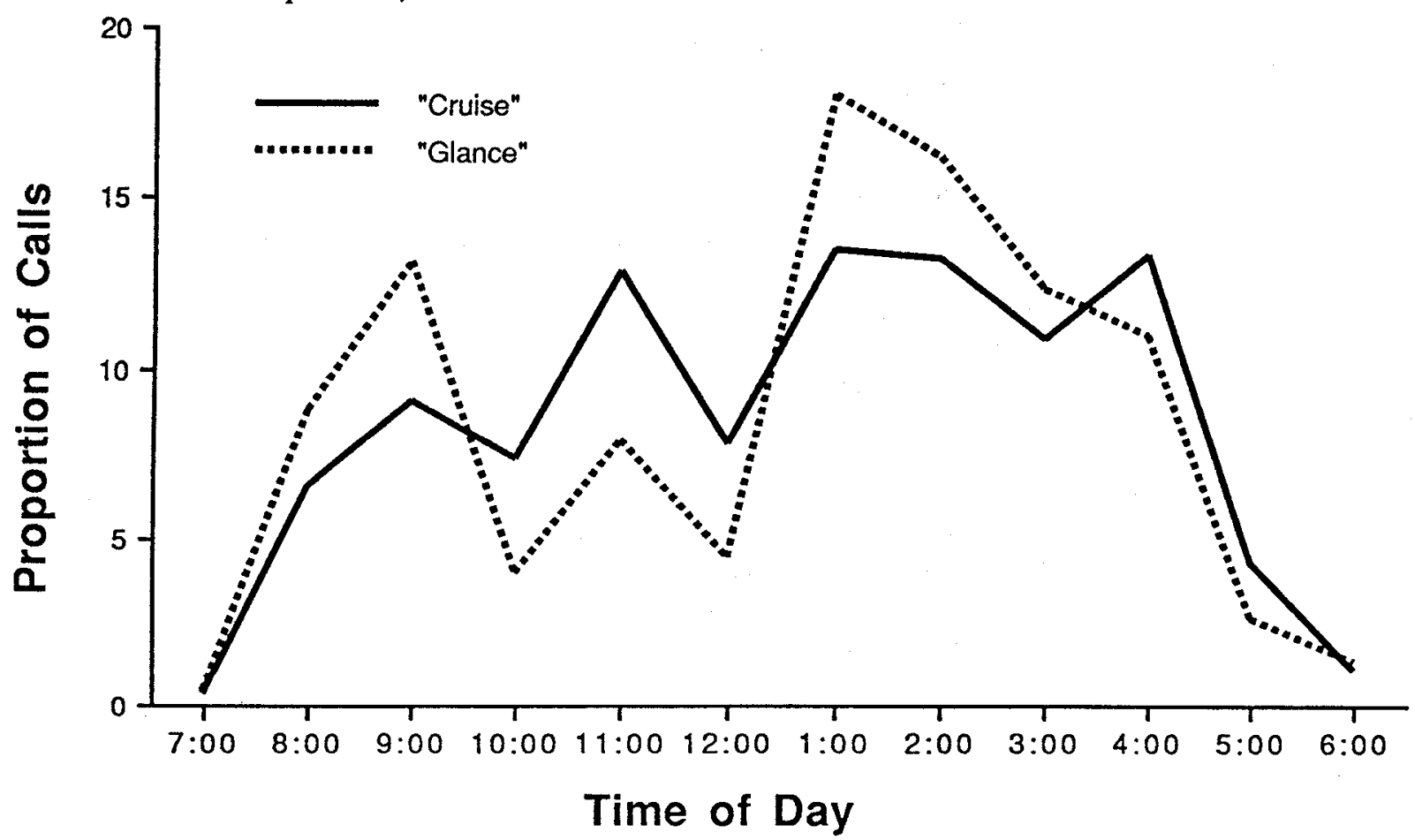

Figure 2. Call attempts by time of day. 
Sometimes the resource needed to continue a conversation was another person. Several respondents complained that while CRUISER communication was appropriate for pairs, it was unsatisfactory for communication in a larger group because there were no satisfactory multiperson conferencing facilities available in it.

Other needs for communication richness occurred when people were dealing with social relationships and ambiguities, rather than work objects. Thus, many of the mentors reported that they used the CRUISER system to inquire about status, but met face-to-face when giving students feedback about their personal performance, for example, after a student presentation. One mentor said he scheduled a face-to-face feedback session because he anticipated a long meeting in which the parties would need a "richer" (his term) communication environment, including the ability to move around and to see and respond to subtle reactions.

These differences in topics of conversation led to differences in the perceived usefulness of CRUISER conversations versus face to face conversations. Respondents reported that CRUISER conversations were less useful, both for getting work done and for learning about their conversational partner as shown at the bottom of Table 2.4 These data were derived by measuring respondents' assessments of a series of 7-point Likert items. These items were then combined into three scales, with item assignment based on a principal components factor analysis with varimax rotation. The scales were: Productivity (e.g., usefulness for getting your work done; relevance to your ongoing work; 4 items, Cronbach's alpha $=.73$ ), Organizational culture (e.g., usefulness for keeping up with company people, politics, policies, and other news; usefulness for providing background information about how things are done at this company; 2 items, Cronbach's alpha $=.75$ ) and Relationship maintenance (e.g., usefulness for maintaining a personal relationship with someone at work; usefulness for understanding your partners point of view; 3 items, Cronbach's alpha $=.53$ )

\footnotetext{
${ }^{4}$ While Table 2 shows paired $t$-tests between CRUISER and face-to-face conversation, the differences between the modalities hold when duration is controlled, primarily because conversation duration was only weakly correlated with the outcome judgments ( $r$ 's between -.14 for the relationship scale to .14 for the productivity scale). Rather than conversational length per se, respondents reported that work was accomplished during a meeting when the topics discussed included solving problems $(\beta=$ $.34, \mathrm{p}<.005)$, making decisions $(\beta=.23, \mathrm{p}<.05)$, and assigning work $(\beta=.28, p<.05)$, but that less work was accomplished when they talked about scheduling work ( $\beta$ $=.-.20, \mathrm{p}<.10 ; \mathrm{R}^{2}$ for the model $=.34$ ).
}

\begin{tabular}{|c|c|c|c|}
\hline \multirow[t]{2}{*}{ Measure } & \multicolumn{2}{|l|}{ Modality } & \multirow[b]{2}{*}{$\begin{array}{l}\text { Paired } \\
\text { t-test }\end{array}$} \\
\hline & CRUISER & $\begin{array}{l}\text { Face } \\
\text { to } \\
\text { face }\end{array}$ & \\
\hline $\begin{array}{l}\text { Conversation length } \\
\text { (in minutes, self-report) }\end{array}$ & 3.9 & 35.7 & $-3.96 * * *$ \\
\hline$\%$ meeting work related & 90 & 89 & .00 \\
\hline \multicolumn{4}{|c|}{$\begin{array}{l}\text { Meeting topic } \\
\text { (\% of number of meetings) }\end{array}$} \\
\hline $\begin{array}{l}\text { Schedule meetings } \\
\text { \& tasks }\end{array}$ & 48 & 19 & 2.03 \\
\hline Assign tasks to people & 14 & 19 & -.57 \\
\hline Report work status & 71 & 81 & -1.00 \\
\hline Solve problems & 48 & 86 & $-2.61^{* *}$ \\
\hline Make decisions & 33 & 57 & -1.75 \\
\hline $\begin{array}{l}\text { Discuss workplace } \\
\text { information }\end{array}$ & 0 & 10 & -1.45 \\
\hline $\begin{array}{l}\text { Discuss nonworkplace } \\
\text { topics }\end{array}$ & 5 & 5 & .00 \\
\hline Greet another & 33 & 19 & 1.83 \\
\hline \multicolumn{4}{|l|}{ Outcomes of meetings } \\
\hline Productive work done & 4.77 & 5.94 & $-3.92 * * *$ \\
\hline \multicolumn{2}{|c|}{ Learn organizational culture 1.88} & 2.10 & -0.81 \\
\hline $\begin{array}{l}\text { Relationship } \\
\text { maintenance }\end{array}$ & 4.29 & 5.19 & $-4.26 * * *$ \\
\hline
\end{tabular}

Table 2. Duration, topics discussed, and outcomes of conversations

\section{Perceptions of media}

People have experience with a wide variety of communications media. When they have a task that requires communication, they choose the medium that seems most appropriate. This choice is not random. Different media have properties that people perceive to be differentially useful in specific situations. For instance, Short, Williams, \& Christie [28] found that the outcome of communications tasks involving interpersonal conflict (e.g., bargaining) and interpersonal relations (e.g., getting to know someone, forming impressions) can be affected by the medium chosen to undertake them.

We asked participants in our study to rate the appropriateness of nine different media for a variety of communications tasks. The media were one-on-one face-to-face meetings, group meetings, telephone, electronic mail, answering machines, fax, handwritten notes, printed documents, and CRUISER desktop videoconferencing. The communications tasks were: asking questions, generating ideas, staying in touch, scheduling meetings, checking on project status, making decisions, exchanging confidential information, explaining a difficult 


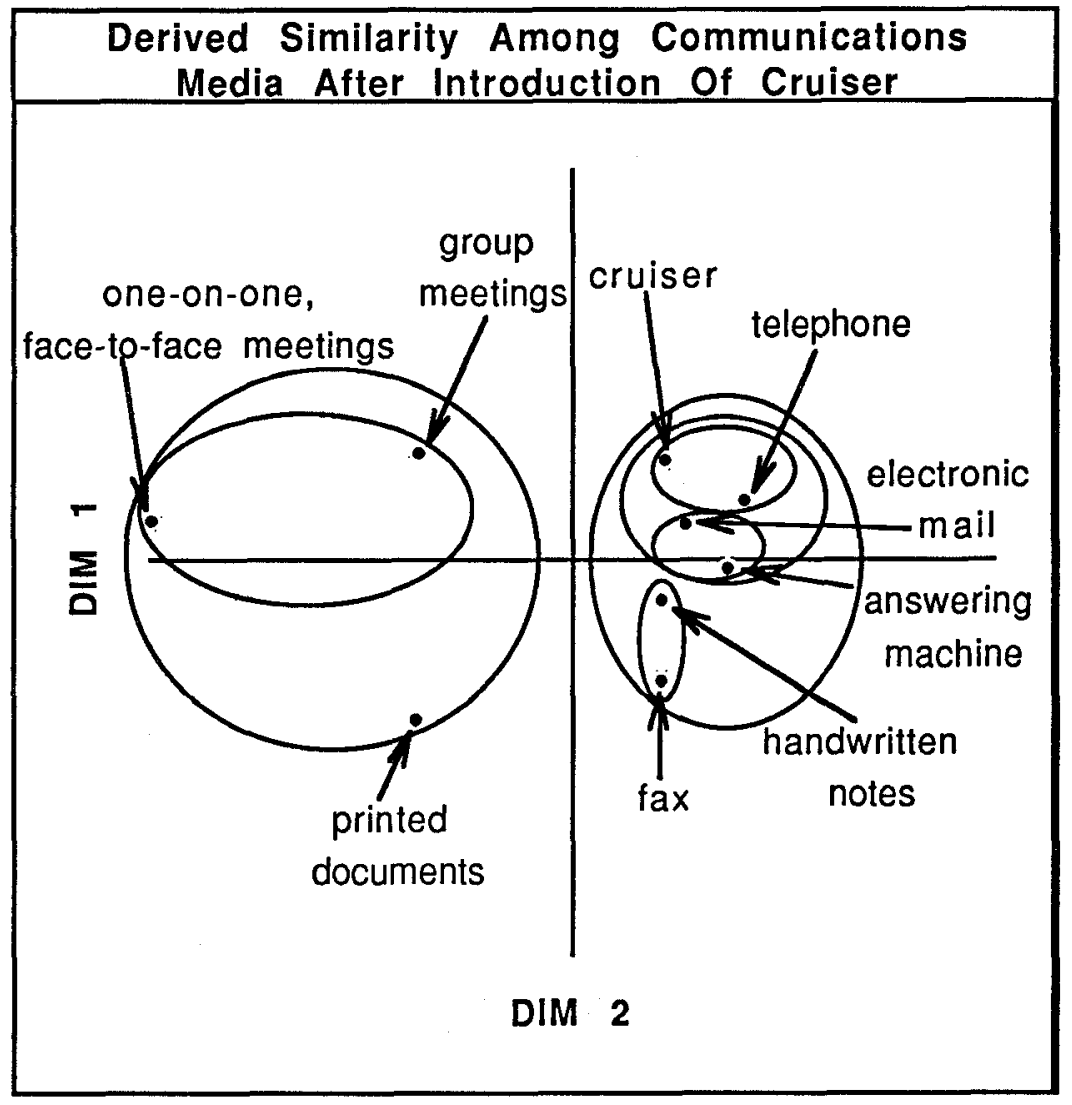

Figure 3. Similarity of communication media.

concept, making commitments, resolving disagreements, exchanging time-sensitive information, negotiating and bargaining, exchanging information, and getting to know someone. This list includes many of the same communication activities that Short et. al. used in their study of the utility of teleconferencing systems [28]. Participants were asked to do this set of ratings twice, once at the start of the study, before they had used the CRUISER system, and once after using the CRUISER system for a month. Because users were not yet familiar with the CRUISER software, the first set of ratings did not include the CRUISER system among the media.

To derive a similarity measure between each of the media we first calculated the mean appropriateness of each medium for each task. We then computed Pearson correlation coefficients between the ratings for each of the media on the 14 communication activities. This resulted in a matrix of 36 pairwise correlations for the ratings collected after the CRUISER system was introduced. This matrix of correlations was treated as similarities and used as input to the KYST [18] nonmetric multidimensional scaling program. This technique $[17,27]$ attempts to create from the similarities a dimensional representation in which distances between points are related to their similarity. In addition, a hierarchical clustering solution was derived for the same sets of data using the methods of Johnson [12].
Figure 3 shows the two dimensional solution (stress $=.008$, Formula 1) from these multidimensional scaling and hierarchical clustering procedures. The vertical dimension can be interpreted as the degree of interactivity that a particular medium provides. For instance, group meetings, electronic mail and the telephone are seen as being highly interactive while fax, handwritten notes and printed documents are perceived as less so. The horizontal dimension can be interpreted as the amount of information exchanged through a medium in a typical communication. Thus, users perceived that in one-on-one face-to-face meetings a great deal of information is transmitted while much less is transmitted in the typical telephone call or answering machine message.

The media on the right side of the plot differ from each other primarily in terms of their interactivity. CRUISER and the telephone are clustered with electronic mail and answering machines, but are more interactive, presumably because they offer real-time communication. CRUISER, in turn, is perceived to be a slightly more interactive and information bearing version of the telephone with which it is clustered. This makes sense, since the CRUISER system adds visual information to the audio information transmitted by the telephone. 
Table 3 shows the mean rating of appropriateness for the same set of communication tasks listed above for the CRUISER system, face-to-face communication, and the telephone. Ratings were judged on a 1 (Inappropriate) to 5 (Appropriate) scale and the tasks are shown below in increasing order of the appropriateness of the CRUISER system for doing them.

\begin{tabular}{|l|l|l|l|}
\hline Task & CRUISER & $\begin{array}{l}\text { Face } \\
\text { to } \\
\text { Face }\end{array}$ & Phone \\
\hline $\begin{array}{l}\text { Exchanging confidential } \\
\text { information }\end{array}$ & 2.66 & 5.00 & 3.50 \\
\hline $\begin{array}{l}\text { Explaining a difficult } \\
\text { concept }\end{array}$ & 3.00 & 5.00 & 2.61 \\
\hline Getting to know someone & 3.14 & 5.00 & 2.68 \\
\hline Resolving disagreements & 3.59 & 5.00 & 3.50 \\
\hline Negotiating, bargaining & 3.68 & 5.00 & 3.64 \\
\hline Generating ideas & 3.82 & 5.00 & 3.39 \\
\hline Making decisions & 4.00 & 4.95 & 3.73 \\
\hline Making commitments & 4.14 & 4.86 & 4.14 \\
\hline Scheduling meetings & 4.18 & 4.41 & 4.50 \\
\hline Exchanging information & 4.27 & 4.77 & 4.14 \\
\hline Asking questions & 4.27 & 5.00 & 4.41 \\
\hline Staying in touch & 4.32 & 4.73 & 4.64 \\
\hline $\begin{array}{l}\text { Exchanging time-sensitive } \\
\text { information }\end{array}$ & 4.41 & 4.23 & 4.68 \\
\hline Checking project status & 4.50 & 4.86 & 4.14 \\
\hline
\end{tabular}

Table 3 Appropriateness of three media for different tasks

Note that both the CRUISER medium and the telephone medium were judged inferior to one-on-one, face-to-face communication for most tasks. The CRUISER system was judged especially useful for checking on project status, keeping in touch, and exchanging information of various types. As the tasks become more socially sensitive or intellectually difficult, both CRUISER and telephone media are judged less adequate, while face-to-face communication is judged more adequate.

Taken together, these data support the conclusion that in this study; the CRUISER service was judged to be useful for similar tasks as the telephone and was perceived as being quite different from face-to-face conversation.

\section{Privacy}

The debriefing interviews asked users about their privacy concerns after four weeks of system use. In contrast to concerns expressed while the system was being designed, most users did not think privacy violations were a problem, especially within a small, collaborative community. Only 4 of the 23 users did not want strangers to have the ability to look into their offices. As we said earlier, mentors and interns described a recent face-to-face and CRUISER conversation with their partner. For each conversation they were asked, "How much did this conversation violate your privacy?" Since people are likely to feel more intruded upon when they are the recipient rather than the initiator of a conversational attempt, the interview ascertained who had started the conversation.

An analysis of variance of these data shows a main effect of initiation $(F=2.85, p<.01)$ i.e., the recipient of a conversational attempt felts more intruded upon than the initiator, but no significant main effect of communication modality, i.e., on average, CRUISER conversations and face-to-face conversations were equally privacy invasive. However, there was a significant interaction between initiation and modality $(\mathrm{F}=2.39$, $\mathrm{p}<.05)$ that showed that the recipients of conversational attempts felt substantially more privacy violation when the attempt was made via CRUISER than when it was made faceto-face. This interaction is shown in Figure 4.

Surprisingly, the privacy of outgoing messages was as large a concern as the fear of others snooping into one's office. Because cameras have only a fixed field of view, typically narrower than the human visual system, users were concerned that other people might be present at the called party's location but invisible when they were having a conversation. They also were aware that the hands-free audio in the CRUISER system meant that others physically in the vicinity of a conversation could overhear it. As a result, employees sometimes held faceto-face meetings when they wanted to insure that they were not disturbing others or that others could not overhear them.

\section{Autocruises}

CRUISER was designed to support informal communication and increase opportunities for contact in a manner analogous to physical proximity. As we have seen, the glance was a mechanism with a reasonable degree of use and success. The autocruise was another mechanism, designed to mimic the opportunities for conversation that people have when they pass by another in the hallway. The autocruise was a systeminitiated call attempt, in which two randomly selected users who were currently logged in were connected using the standard cruise protocol (i.e., a several second connection that timed-out if neither party accepted the call).

The probability of accepting an autocruise was substantially less than accepting a human-initiated CRUISER call. Of the 236 autocruises conducted during business hours, from $8 \mathrm{AM}$ to 5PM, about $3 \%$ were accepted compared to $54 \%$ of userinitiated calls. This $3 \%$ includes only those calls that were converted to a more substantial conversation and does not include the many acknowledgements, greetings and other brief exchanges that occurred during the several second interval before the autocruise timed-out. 


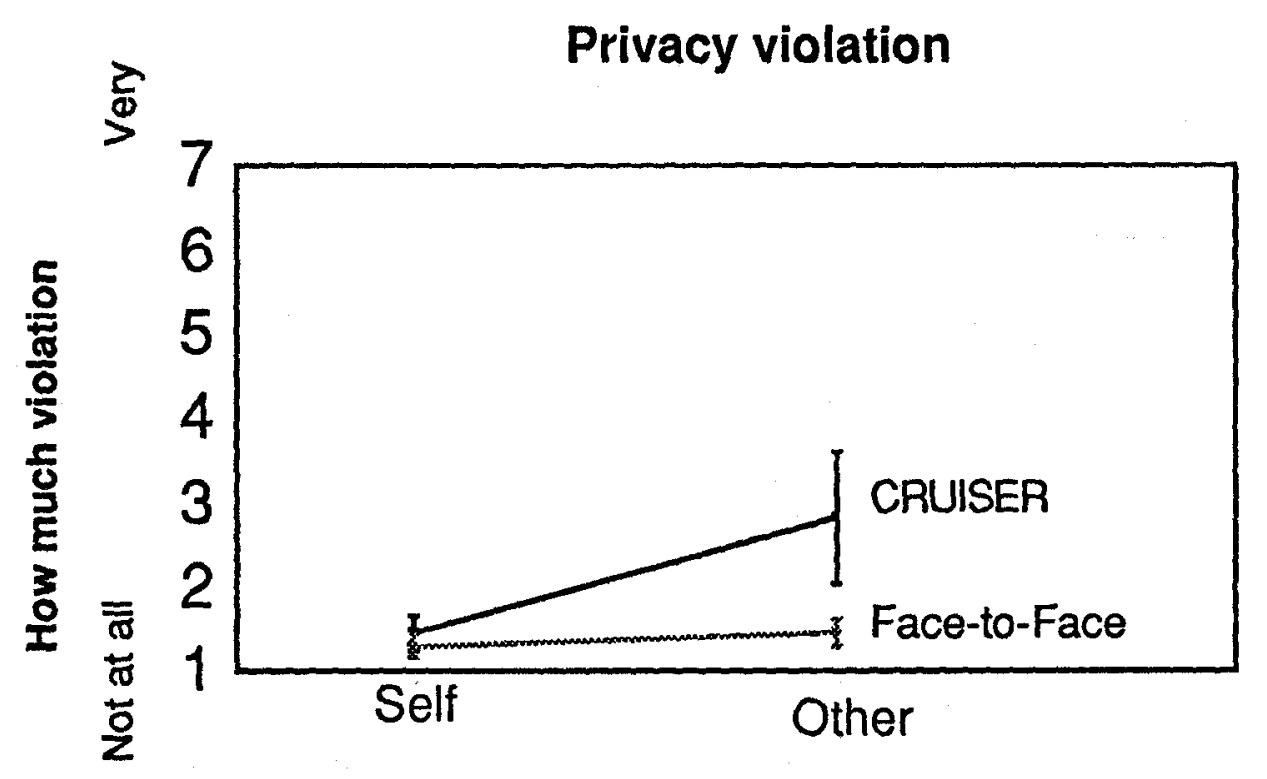

Who started the conversation

Figure 4. Perceptions of privacy violation.

There are multiple reasons for the low conversation rate for autocruises. For an autocruise to be accepted two parties need to be in their offices, they need to notice that a call attempt occurred, and they need to want to talk to the potential partner at just the moment that the conversational opportunity presents itself. The combination of these probabilities is probably the reason that few autocruises were converted into conversations. In addition, our results suggest that users found autocruises highly disconcerting. When asked to describe features of the CRUISER system they disliked most, $40 \%$ of users mentioned autocruises.

\section{DISCUSSION}

\section{DId we succeed?}

Were we able to show the value of desktop videoconferencing for informal communication? If we use the criteria of frequency, expressiveness, and interactivity as the hallmarks of informal communication that we would like see in the use of a new medium, how did we do with respect to these criteria? The CRUISER system was certainly used a lot, with a frequency equal to or exceeding that of a long established and much more ubiquitous medium like the telephone. This indicates that the system was convenient to use. It also provides evidence for the prediction that desktop videoconferencing will be used more frequently than special-purpose videoteleconferencing facilities because desktop placement reduces the behavioral cost of gaining access to the technology [9].
Expressivity, however, was not as well served. The short call length, its use for scheduling and status reporting rather than problem solving or decision making, and its perceived similarity to other media where the amount of information typically transmitted is limited all indicate that there were many expressive functions for which users did not find the CRUISER system sufficiently suitable. This result is probably related to the lack of support for access to other tools and artifacts (e.g. shared blackboards and editors, etc.) within the context of the conversation and the lack of support within the version of the CRUISER system tested here for multiparty conversations.

In terms of interactivity, we had thought that because we supported both a high quality full-duplex audio channel and a high quality, full-motion video channel that this would be sufficient. It wasn't. Although conversations, once started, seemed quite normal and interactive and the use of the glance mechanism showed that the system was used for some of the exploratory behaviors that are necessary to maintain awareness of possible conversational partners, other features designed to promote the interactivity of informal communication were not successful. The infrequent completion of autocruise calls, the perception of the autocruise mechanism as intrusive, and users' judgments that receiving CRUISER calls was more privacy invading than face to face interactions, all indicate that the implementation of the visual channel mechanism that allowed users to assess, negotiate, start, and end a conversation was not good enough. The specific conversational protocol that people 
use for informal face to face communication was not able to operate and it was not replaced by something that was sufficiently functional.

Hence, while the version of the CRUISER system we tested here had some successes, it by no means achieved the degree of support for informal communication that we would have liked. For the most part people perceived it and used it like a telephone or an electronic mail system; they adopted the new technology to old uses.

\section{What happened and why?}

Perhaps the most important failure of the system was that it inadequately provided users the resources they needed to ease into communication. In the workaday world, when people pass each other in the corridor or pass by another's office, they have subtle, but well practiced mechanisms to assess or signal readiness for communication and to manage the transitions between lack of engagement to engagement and from engagement to disengagement $[13,15]$. If one party does not wish to communicate with another, he or she can use lack of eye contact and other nonverbal displays to signal this, which in turn often aborts the conversational attempt. The potential initiator can assess another's engagement in a task without the other being aware that an assessment took place. In these cases, the failure to hold a conversation is a cooperative act, in which neither party is explicitly rejected by the other.

In contrast, the conversation opportunity mechanism we used was abrupt, intrusive, and lacking in subtlety. A conversational opportunity was announced when a potential conversational partner instantaneously showed up on one's screen already at speaking distance. Because the screen filled with an image with sudden onset (and the call was frequently announced with a ring as well), the presence of the other was often highly intrusive. Converting an opportunity into a conversation required explicit and one-sided action, when one party to a call issued the "visit" command. These conditions placed too much pressure on the parties to acknowledge each other before they had conducted any negotiation about whether to have a conversation. Users complained that being suddenly confronted with another compelled conversation, even when they didn't want one. In these circumstances, a failure to have the conversation became a explicit rejection, as well. As one user described it, "There is no half way with CRUISER."

To support the expressivity dimension of informal communication, communication tools for casual conversation must also support sustained task-focused work and must allow graceful transition to it. Users repeatedly described attempting to use the occasion of running into an appropriate partner as an opportunity to seek help for a current problem. They were stymied because they couldn't illustrate their dilemma with diagrams or share the object that was vexing them and this contributed to the brevity of their conversations.
The traditional uses to which CRUISER and other desktop teleconference systems have been put and perceptions of their usefulness may also reflect their novelty in several other ways. First, because new communication systems rarely have a critical mass of users at the start, they are indeed less useful than more entrenched systems. During the experiment period, users could only contact 22 other people using CRUISER, several of whom dropped out of the trial and over half of whom they didn't know well enough to initiate even a single call. Second, when new technologies are introduced, people often judge them by the standards of the current world, being critical of the ways they violate the current order without appreciating the new opportunities they allow. Thus, Perry [23] notes that in its early days, telephony, particularly in Great Britain, was thought of as an impolite, privacy invasive medium. Only later did other attributes -- the ability to screen visitors, to reduce loneliness, to summon help in emergencies, and to increase convenience, for example -- become part of the public discourse about the telephone. Third, as technologies mature, both new uses and new norms about use develop, so that over time the technology and how it is used change. New uses that extend beyond standard telephony -- for example, the virtual shared office and orientation glances -- were starting to develop over the course of the experiment.. In summary, while the data presented here suggest that during the trial, the dominant use of the CRUISER system was as a visual telephone used for checking status, asking questions, and passing brief messages it is hard to predict what later use will be from these early experiences.

\section{What will we try next?}

Our experience with the CRUISER system leads us to believe that supporting informal communication will require managing some of the tensions and transitions in human behavior. We must balance the costs of providing opportunities for communication with the benefit of any particular opportunity. To provide these opportunities we must balance the tensions between accessibility, privacy and solitude. Moreover, from the point of view of technology design, we must develop better mechanisms to support the transitions between conversational states that people easily manage in their workaday world -- the transitions between non-engagement and engagement, between engagement and disengagement, and between casual conversation and work.

Communication technology to support informal interaction must provide light weight opportunities for interaction. In social interaction, a light weight opportunity would be one in which getting into a preconversational attitude is a side effect of other activity and thus allows conversation with little incremental effort. From the preconversational state, potential interactants, by small adjustments and subtle cues, can cooperatively determine whether an acknowledgement, greeting, conversation, or nothing will take place. The failure of the 
relatively gross techniques that we tried suggests some of the subtlety of everyday conversational coordination and suggests that substantial ingenuity will be required to provide analogous mechanisms in a synthetic world.

Balancing the three factors of accessibility, privacy, and solitude is a must. Accessibility is the ability of one individual to have easy access to another. This is one of essential properties of informal communication. Privacy is the ability of an individual to control the information about him or herself available to others. And solitude is the ability of an individual to control others' intrusion into his or her space or consumption of his or her time. Individuals would like to have all three, but at the level of the group, they are incompatible. Having access to other people at a convenient time often violates their solitude. The use and abuse of telephones, open offices, and private secretaries has shown that even in conventional environments achieving this balance is neither automatic nor static.

Finally, managing the transition between pure conversation and doing work will require the integration of conversational props [5]. Conversational props are the artifacts and resources needed to sustain group work. For these props to be used spontaneously in the support of ongoing conversation, they must be easily and quickly accessible during the course of the conversation and they must be easily shared. All members of the conversation must be able to view, point at, and if appropriate, modify objects outside the conversation itself data, diagrams, and files, for example.

\section{CONCLUSIONS}

Based on our own intuitions and the literature on informal communication we have speculated that some form of desktop videoteleconferencing could prove useful in preserving informal communication channels for geographically distributed organizations. We've presented in this paper some results from our initial attempt to prototype such a system. While our results indicated that we were able to produce a system whose frequency of use conformed to our expectations, there was substantial room for improvement in the system along the dimensions of expressivity and interactivity. Some of the necessary improvement will come as users develop experience with these sort of systems and some will come through the sort of iterative technology improvements we've outlined above. We remain relatively confident, however, that technologies of this sort remain the best hope for providing an informal communications mechanism that will lead to successful and productive distributed organizations.

\section{ACKNOWLEDGMENTS}

Robert Fish, Robert Kraut and Robert Root designed the CRUISER application, and Richard Clayton and Robert Root programmed it. Bill Campbell, Chris Colasante, Jennifer Murray, and Mark Shifflet provided critical assistance in setting up the network, assisting users, and gathering and analyzing data. Tom Judd and David Braun provided help in hardware and software design, respectively.

\section{REFERENCES}

1. Abel, M.J. (1990). Experiences in an exploratory distributed organization. In J. Galegher \& R. Kraut (Eds.), Intellectual teamwork: Social and technological foundations of group work. Hillsdale, NJ: Lawrence Erlbaum Associates.

2. Altman, S., Valenzi, E. \& Hodgetts, R. (1985) Organizational behavior: Theory and practice. Orlando, Florida: Academic Press.

3. Borning, A. \& Travers, M. (1991) Two approaches to casual interaction over computer and video networks. In Proceedings of CHI '91, Conference on Human Factors in Computing Systems. (New Orleans, Louisiana) Association for Computing Machinery, New York.

4. Bulick, S., Abel, M., Corey, D., Schmidt, J. \& Coffin, S. (1989) The U.S.West Advanced Technologies prototype multi-media communications system. Globecom'89: Proceedings of the IEEE Global Telecommunications Conference. Dallas, Texas.

5. Cruz, G.C., Gomez, L.M., \& Wilner, W.T. (1991) Tools to support conversational multimedia. In Globecom '91, Proceedings of the IEEE Global Telecommunications Conference, Phoenix, Arizona.

6. Daft, R. L., \& Lengel, R. H. (1984). Information richness: A new approach to managerial behavior and organization design. In B. Staw \& L. L. Cummings (Eds.), Research in organizational behavior (Vol. 6). Greenwich, CT: JAI Press.

7. Daft, R. L. \& Lengel, R. H. (1986) Organizational information requirements, media richness, and structural design. Management Science, 32, 554-571.

8. Fish, R. S. (1989) CRUISER: A multimedia system for social browsing. Siggraph Video Review, Vol 45, No. 6 New York: Association for Computing Machinery

9. Fish, R.S., Kraut, R.E., \& Chalfonte, B.L. The VideoWindow system in informal communications. In Proceedings of the Conference on Computer Supported Cooperative Work (CSCW '90), pp. 1-11, Los Angeles, California, ACM Press. 
10. Goodman, G. O. \& Abel, M. J. (1987) Communication and collaboration: Facilitating cooperative work through communication. Office: Technology and People, 3, 129-146.

11. Heath, C. \& Luff, P. (1991) Disembodied conduct: Communication through video in a multi-media office environment. In Proceedings of $\mathrm{CHI}$ '9l, Conference on Human Factors in Computing Systems. (New Orleans, Louisiana) Association for Computing Machinery, New York.

12. Johnson, S.C. Hierarchical clustering schemes. Psychometrika, 32, 241-254.

13. Kendon, A. \& Ferber, A. (1973). A description of some human greetings. In R. Michael \& J. Crook (Eds.), Comparative ecology and behavior of primates. London: Academic Press.

14. Kiesler, S., Siegel, J. \& McGuire, T. (1984). Social psychological aspects of computer-mediated communication. American Psychologist, 39, 11231134.

15. Knapp, Mark L.(1978). Social intercourse: From greeting to goodbye. Boston: Allyn \& Bacon.

16. Kraut, R.E., Fish, R.S., Root, R.W., \& Chalfonte, B.L. (1990). Informal communication in organizations: Form, function, and technology. In S. Oskamp \& S. Spacapan (Eds)., Human Reactions to Technology: The Claremont Symposium on Applied Social Psychology. Beverly Hills, CA: Sage Publications. Pp 145-199.

17. Kruskal, J. (1964) Multidimensional scaling by optimizing goodness of fit to a nonmetric hypothesis. Psychometrika, 29, 1-27.

18. Kruskal, J., Young, F. \& Seery, J. (1977) How to use KYST- $2 \mathrm{~A}$, a very flexible program to do multidimensional scaling and unfolding. Unpublished paper, Bell Laboratories

19. Louie, G., Mantei, M., \& Buxton, W.A.S. (1991) Making contact in a multi-media environment. $\mathrm{HCI}$ Consortium on CSCW, Ann Arbor, MI., February, 1991.
20. Mayer, M. (1977). The telephone and the uses of time. In I. de Sola Pool (Ed.) The social impact of the telephone. Cambridge, Massachusetts: MIT Press. Pp 225-245.

21. Monge, P. R., Rothman, L. W., Eisenberg, E. M. , Miller, K. L. \& Kirste, K. K. (1985). The dynamics of organizational proximity. Management Science 31, 1129-1141.

22. Olson, M. \& Bly, S. (1991). The Portland experience: A report on a distributed research group. International Journal of Man-Machine Studies, 34, 211-228.

23. Perry, C. R. (1977) The British experience 18761912: The impact of the telephone during the years of delay. In I. de Sola Pool (Ed.) The social impact of the telephone. Cambridge, Massachusetts: MIT Press.

24. Rockoff, Maxine (1975a). An overview of some technological/heath-care system implications of seven exploratory broad-band communication experiments. IEEE Transactions on Communications, COM-23 , 20-30.

25. Rockoff, Maxine (1975b). The social implications of health care communication systems. IEEE Transactions on Communications, COM-23 (10), 1085-1088.

26. Root, R.W. (1988). Design of a multi-media system for social browsing. Proceedings of the Conference on Computer-Supported Cooperative Work. Portland, OR, 25-38.

27. Shepard, R. N. (1962) The analysis of proximities: Multidimensional scaling with an unknown distance function. Psychometrika, 27,127-140, 219-246.

28. Short, J., Williams, E., \& Christie, B. (1976) The social psychology of telecommunications. London: John Wiley \& Sons.

29. Stohl, C. \& Redding, W. C. (1987). Messages and message exchange processes. In J. Jablin, L. Putnam, K. Roberts, \& L. Porter (Eds.), Handbook of Organizational Communication. Newbury Park, CA: Sage Publications. Pp. 451-502. 